

\title{
SEMICLASSICAL TRACE FORMULAS AND HEAT EXPANSIONS
}

\author{
Yves Colin DE VeRdière
}

In a recent paper (J. Phys. A 43:47 (2011), 474028), B. Helffer and R. Purice compute the second term of a semiclassical trace formula for a Schrödinger operator with magnetic field. We show how to recover their formula by using the methods developed by Riemannian geometers in the seventies for heat expansions.

\section{Introduction}

There is a strong similarity between the expansions of the heat kernel as worked out by people in Riemannian geometry in the seventies, starting with the famous "Can one hear the shape of a drum?" by Mark Kac [1966] and continuing with [Berger 1966; McKean and Singer 1967] (see also the books [Berger et al. 1971; Gilkey 1975]), and the so-called semiclassical trace formulas developed by people in semiclassical analysis, starting with [Helffer and Robert 1983]. In fact, this is not only a similarity, but, as we will prove, each of these expansions, even if they differ when expressed numerically for some example, can be deduced from the other one as formal expressions of the fields.

Let us look first at the heat expansion on a smooth closed Riemannian manifold of dimension $d,(X, g)$, with the (negative) Laplacian $\Delta_{g}{ }^{1}$. The heat kernel $e(t, x, y)$, with $t>0$ and $x, y \in X$, is the Schwartz kernel of $\exp \left(t \Delta_{g}\right)$ : the solution of the heat equation $u_{t}-\Delta_{g} u=0$ with initial datum $u_{0}$ is given by

$$
u(t, x)=\int_{X} e(t, x, y) u_{0}(y)|d y|_{g} .
$$

The function $e(t, x, x)$ admits, as $t \rightarrow 0^{+}$, the following asymptotic expansion:

$$
e(t, x, x) \sim(4 \pi t)^{-d / 2}\left(1+a_{1}(x) t+\cdots+a_{l}(x) t^{l}+\cdots\right) .
$$

The $a_{l}$ are given explicitly in [Gilkey 2004, p. 201] for $l \leq 3$, and are known for $l \leq 5$ [Avramidi 1990; Ven 1998]. See also the related works [Hitrik 2002; Hitrik and Polterovich 2003a; 2003b; Polterovich 2000]. They are universal polynomials in the components of the curvature tensor and its covariant derivatives. For example, $a_{0}=1$ and $a_{1}=\tau_{g} / 6$, where $\tau_{g}$ is the scalar curvature.

The previous asymptotic expansion gives the expansion of the trace by integration over $X$ and has been used as an important tool in spectral geometry:

$$
\operatorname{trace}\left(e^{t \Delta_{g}}\right)=\int_{X} e(t, x, x)|d x|_{g}=\sum_{k=1}^{\infty} e^{\lambda_{k} t},
$$

MSC2010: 35P20, 35S05, 58J35.

Keywords: trace formula, magnetic fields, heat expansion, synchronous gauge.

${ }^{1}$ In this note, we will not follow the usual sign convention of geometers, but the convention of analysts 
where $-\lambda_{1}=0 \leq-\lambda_{2} \leq \cdots \leq-\lambda_{k} \leq \cdots$ is the sequence of eigenvalues of $-\Delta_{g}$ with the usual convention about multiplicities. If $d=2$, this gives

$$
\operatorname{trace}\left(e^{t \Delta_{g}}\right)=\frac{1}{4 \pi t}\left(\operatorname{Area}(X)+\frac{2 \pi \chi(X)}{6} t+O\left(t^{2}\right)\right)
$$

where $\chi(X)$ is the Euler characteristic of $X$.

There is an extension of the previous expansion in the case of Laplace type operators on fiber bundles: the coefficients of the expansion are then polynomials in the covariant derivatives of the curvature of the metric and of the connection on the fiber bundle. The heat expansion can be reinterpreted as an expansion of the Schwartz kernel of $f\left(-\hbar^{2} \Delta_{g}\right)$ on the diagonal $x=y$ in powers of $\hbar$ with $f(u)=\exp (-u)$ and $t=\hbar^{2}$. This is a particular case of the semiclassical trace.

Let us describe the semiclassical setting in the flat case: $\widehat{H}_{\hbar}$ is a self-adjoint $\hbar$-pseudodifferential operator with Weyl symbol $H(x, \xi)$ in some open domain $X$ in $\mathbb{R}^{d}$, or more generally on a Riemannian manifold. Let $f \in \mathscr{S}(\mathbb{R})$ and look at $f\left(\hat{H}_{\hbar}\right)$. Under some suitable assumptions (ellipticity at infinity in $\xi$ ) on $H, f\left(\hat{H}_{\hbar}\right)$ is a pseudodifferential operator whose Weyl symbol $f^{\star}(H)$ is a formal power series in $\hbar$, given, using the Moyal product denoted by $\star$, by the following formula (see [Gracia-Saz 2005] for explicit formulas and Section 4.2 therein for a proof; see also [Charles 2003]) at the point $z_{0} \in T^{\star} X$ :

$$
f^{\star}(H)\left(z_{0}\right)=(2 \pi \hbar)^{-d} \sum_{l=0}^{\infty} \frac{1}{l !} f^{(l)}\left(H\left(z_{0}\right)\right)\left(H-H\left(z_{0}\right)\right)^{\star l}\left(z_{0}\right) .
$$

From the previous formula, we see that the symbol of $f\left(\hat{H}_{\hbar}\right)$ at the point $z$ depends only of the Taylor expansions of $H$ at the point $z$ and of $f$ at the point $H(z)$. Helffer and Purice [2010] have studied the case of the magnetic Schrödinger operator whose Weyl symbol is $H_{a, V}(x, \xi)=\sum_{j=1}^{d}\left(\xi_{j}-a_{j}(x)\right)^{2}+V(x)$ and show that the Schwartz kernel of $f\left(\hat{H}_{\hbar, a, V}\right)$ at the point $(x, x)$ admits an asymptotic expansion of the form

$$
\left[f\left(\hat{H}_{\hbar, a, V}\right)\right](x, x)=(2 \pi \hbar)^{-d} \sum_{j=0}^{\infty} \hbar^{2 j}\left(\sum_{l=0}^{k_{j}} \int_{\mathbb{R}^{d}} f^{(l)}\left(\|\xi\|^{2}+V(x)\right) Q_{j, l}^{a, V}(x, \xi)|d \xi|\right)
$$

where the $Q_{j, l}^{a, V}(x, \xi)$ are polynomials in $\xi$ calculated from the Taylor expansions of the magnetic field $B=d a$ and $V$ at the point $x$. The proof in [Helffer and Purice 2010] uses a pseudodifferential calculus adapted to the magnetic field.

We will give a simplified version of the expansion replacing the (non-unique) $Q_{j, l}^{a, V}(x, \xi)$ by functions $P_{j, l}^{B, V}(x)$ which are uniquely defined and are given by universal $O(d)$-invariant polynomials of the Taylor expansions of $B$ and $V$ at the point $x$. We present then two ways to compute the $P_{j, l}^{B, V}$ :

- we can first use Weyl's invariant theory (see [Gilkey 2004]) in order to reduce the problem to the determination of a finite number of numerical coefficients; then simple examples, like harmonic oscillator and constant magnetic field, allow to determine (part of) these coefficients. 
- The $P_{j, l}^{B, V}$ are related in a very simple way to the coefficients of the heat expansion; it is possible to compute the $P_{j, l}^{B, V}$ from the knowledge of the $a_{l}$ for $j+1 \leq l \leq 3 j$. This is enough to recompute the coefficient of $\hbar^{2}$ and also, in principle, the coefficients of $\hbar^{4}$ in the expansion, because the $a_{l}$ are known up to $l=6$ in the case of a flat metric (see [Ven 1998]).

In this note, we will first describe precisely the semiclassical expansion for Schrödinger operators (in the case of an Euclidean metric) and the properties of the functions $P_{j, l}^{B, V}(x)$. Then, we will show how to compute the $P_{j, l}^{B, V}(x)$ using an adaptation of the method used for the heat kernel (Weyl's theorem on invariants and explicit examples). Finally, we will explain how the $a_{l}$ are related to the $P_{j, l}^{B, V}(x)$. This gives us two proofs of the main formula given in [Helffer and Purice 2010]; this paper was the initial motivation to this work.

\section{Semiclassical trace for Schrödinger operators}

In what follows, $X$ is an open domain in $\mathbb{R}^{d}$, equipped with the canonical Euclidean metric, and $\Omega^{k}(X)$ will denote the space of smooth exterior differential forms in $X$. Let us give a Schrödinger operator, with a smooth magnetic field $B=\sum_{1 \leq i<j \leq d} b_{i j} d x_{i} \wedge d x_{j}$ (a closed real 2-form) and a smooth electric potential $V$ (a real-valued smooth function) in $X$. We assume that $V$ is bounded from below. We will assume also that the 2-form $B$ is exact and can be written $B=d a$ and we introduce the Schrödinger operator defined by

$$
H_{\hbar, a, V}=\sum_{j=1}^{d}\left(\frac{\hbar}{i} \frac{\partial}{\partial x_{j}}-a_{j}(x)\right)^{2}+V(x) .
$$

The Weyl symbol of $H_{\hbar, a, V}$ is $H_{a, V}(x, \xi)=\|\xi-a(x)\|^{2}+V(x)$. We denote by $\widehat{H}_{\hbar, a, V}$ a self-adjoint extension of $H_{\hbar, a, V}$ in $L^{2}(X,|d x|)$. Let us give $f \in \mathscr{Y}(\mathbb{R})$ and $\phi \in C_{o}^{\infty}(X)$ and consider the trace of $\phi f\left(\hat{H}_{\hbar, a, V}\right)$ as a distribution on $X \times \mathbb{R}$ (the density of states):

$$
\operatorname{Trace}\left(\phi f\left(\hat{H}_{\hbar, a, V}\right)\right)=\int_{X} Z_{\hbar, a, V}(g)(x) \phi(x)|d x|,
$$

where $Z_{\hbar, a, V}(g)(x)$ is the value at the point $(x, x)$ of the Schwartz kernel of $f\left(\hat{H}_{\hbar, a, V}\right)$.

Theorem 1. We have the following asymptotic expansion in powers of $\hbar$ :

$$
\begin{aligned}
& Z_{\hbar, a, V}(g)(x) \sim \\
& \quad(2 \pi \hbar)^{-d}\left[\int_{\mathbb{R}^{d}} f\left(\|\xi\|^{2}+V(x)\right)|d \xi|+\sum_{j=1}^{\infty} \hbar^{2 j}\left(\sum_{l=j+1}^{l=3 j} P_{j, l}^{B, V}(x) \int_{\mathbb{R}^{d}} f^{(l)}\left(\|\xi\|^{2}+V(x)\right)|d \xi|\right)\right] .
\end{aligned}
$$

We have the explicit formulas

$$
\begin{aligned}
& P_{1,2}^{B, V}=-\frac{1}{6}\left(\Delta V+\|B\|^{2}\right), \quad P_{1,3}^{B, V}=-\frac{1}{12}\|\nabla V\|^{2}, \\
& P_{2,3}^{B, V}=-\frac{1}{180}\left(8\|\nabla B\|^{2}+\left\|d^{\star} B\right\|^{2}+12\langle\Delta B \mid B\rangle+3 \Delta^{2} V\right) .
\end{aligned}
$$


Here $\|B\|^{2}=\sum_{1 \leq i<j \leq d} b_{i j}^{2}, d^{\star}: \Omega^{2}(X) \rightarrow \Omega^{1}(X)$ is the formal adjoint of $d$ used in the definition of the Hodge Laplacian on exterior forms. If $d=3,\|B\|$ is the Euclidean norm of the vector field associated to $B$.

The $P_{j, l}^{B, V}(x)$ are polynomials of the derivatives of $B$ and $V$ at the point $x$. Moreover, if $\lambda, \mu, c$ are constants and we define $\lambda^{\star}(f)(x)=f(\lambda x)$, we have the following scaling properties:

(1) $P_{j, l}^{\lambda \cdot \lambda^{\star}(B), \lambda^{\star}(V)}(x)=\lambda^{2 j} P_{j, l}^{B, V}(\lambda x)$. This will be used with $x=0$.

(2) $P_{j, l}^{\mu B, \mu^{2} V}(x)=\mu^{2(l-j)} P_{j, l}^{B, V}(x)$.

(3) $P_{j, l}^{B, V+c}(x)=P_{j, l}^{B, V}(x)$.

(4) $P_{j, l}^{-B, V}(x)=P_{j, l}^{B, V}(x)$.

(5) The $P_{j, l}^{B, V}$ are invariant by the natural action of the orthogonal group $O(d)$ on the Taylor expansions of $B$ and $V$ at the point $x$.

Remark 1. From the statement of the theorem, we see that the expansion of the density of states is independent of the chosen self-adjoint extension.

As a consequence, we can get the following full trace expansion under some more assumptions:

Corollary 1. Let us assume that $E_{0}=\inf V<E_{\infty}=\liminf _{x \rightarrow \partial X} V(x)$ and that we have chosen the Dirichlet boundary conditions. Let $f \in C_{o}^{\infty}(]-\infty, E_{\infty}[)$, then the trace of $f\left(\widehat{H}_{\hbar, a, V}\right)$ admits the asymptotic expansion

$$
\begin{gathered}
\operatorname{Trace}\left(f\left(\hat{H}_{\hbar, a, V}\right)\right) \sim(2 \pi \hbar)^{-d} \int_{X}\left(\int_{\mathbb{R}^{d}} f\left(\|\xi\|^{2}+V(x)\right)|d \xi|+\cdots\right. \\
\left.\cdots \sum_{j=1}^{\infty} \hbar^{2 j} \sum_{l=j+1}^{l=3 j} P_{j, l}^{B, V}(x) \int_{\mathbb{R}^{d}} f^{(l)}\left(\|\xi\|^{2}+V(x)\right)|d \xi|\right)|d x| .
\end{gathered}
$$

The coefficient of $\hbar^{2}$ can be written as

$$
-\frac{1}{12} \int_{X \times \mathbb{R}^{d}} f^{(2)}\left(\|\xi\|^{2}+V(x)\right)\left(\Delta V(x)+2\|B(x)\|^{2}\right)|d x d \xi| .
$$

The expansion follows from [Helffer and Robert 1983]. An integration by part in $x$ gives

$$
\int_{X} f^{(3)}\left(\|\xi\|^{2}+V(x)\right)\|\nabla V(x)\|^{2}|d x|=-\int_{X} f^{(2)}\left(\|\xi\|^{2}+V(x)\right) \Delta V(x)|d x| .
$$

\section{Existence of the $\hbar$-expansion of $Z_{\hbar, a, V}$}

Using Theorem 2 in the Appendix, we can work in $\mathbb{R}^{d}$ with $a$ and $V$ compactly supported. The existence of the expansion is known in general from [Helffer and Robert 1983] and the calculus of the symbol of 
$f\left(\hat{H}_{\hbar, a, V}\right)$. We get

$$
\int_{X} Z_{\hbar, a, V}(f)(x) \phi(x)|d x|=(2 \pi \hbar)^{-d} \sum_{j=0}^{\infty} \hbar^{2 j} \sum_{l=0}^{k_{j}} \int \phi(x) f^{(l)}\left(H_{a, V}(x, \xi)\right) Q_{j, l}(x, \xi)|d x d \xi|
$$

where the $Q_{j, l}(x, \xi)$ are polynomials in the Taylor expansion of $H_{a, V}$ at the point $(x, \xi)$. The previous expansion is valid for any (admissible) pseudodifferential operator. In the case of Schrödinger operators we can make integrations by part in the integrals $\int f^{(l)}\left(H_{a, V}(x, \xi)\right) Q_{j, l}(x, \xi)|d \xi|$ which reduces to a similar formula where we can replace the $Q_{j, l}(x, \xi)$ by the $P_{j, l}(x)$. This is based on the expansion of $Q_{j, l}$ as a polynomial in $\xi$ in powers of $(\xi-a)$ : odd powers give 0 and even powers can be reduced using

$$
d_{\xi}\left(\left(\xi_{j}-a_{j}\right) f^{(l)}\left(H_{a, V}\right) \iota\left(\partial_{\xi_{j}}\right) d \xi\right)=2\left\|\xi_{j}-a_{j}\right\|^{2} f^{(l+1)}\left(H_{a, V}\right) d \xi+f^{(l)}\left(H_{a, V}\right) d \xi .
$$

We have only to check that the powers of $\xi$ in $Q_{j, l}(x, \xi)$ are less than $l$ : this is based on Equation (1). The coefficients of the $l$-th Moyal power of $H_{a, V}(z)-H_{a, V}\left(z_{0}\right)$ are homogeneous polynomials of degree $l$ in the derivatives of $H_{a, V}(z)$. At the point $z=z_{0}$ only derivatives of order $\geq 1$ are involved. They are all of degree $\leq 1 \mathrm{in} \xi$. Using gauge invariance at the point $x$ (Section 3), we can assume that $a(x)=0$.

\section{Gauge invariance}

If $S: X \rightarrow \mathbb{R}$ is a smooth function, we have

$$
\operatorname{Trace}\left(\phi e^{-i S(x) / \hbar} f\left(\widehat{H}_{\hbar, a, V}\right) e^{i S(x) / \hbar}\right)=\operatorname{Trace}\left(\phi f\left(\widehat{H}_{\hbar, a, V}\right)\right)
$$

and

$$
e^{-i S(x) / \hbar} f\left(\hat{H}_{\hbar, a, V}\right) e^{i S(x) / \hbar}=f\left(\hat{H}_{a+d S, V}\right) .
$$

Hence, we can chose any local gauge $a$ in order to compute the expansion: using the synchronous gauge (see Section 4), we get the individual terms

$$
\int f^{(l)}\left(H_{0, V}\right) P_{j, l}^{B, V}(x)|d \xi|
$$

for the expansion, where the $P_{j, l}^{B, V}(x)$ depend only of the Taylor expansions of $B$ and $V$ at the point $x$.

\section{The synchronous gauge}

The main idea is to find an appropriate gauge $a$ adapted to the point $x_{0}$ where we want to make the symbolic computation. In a geometric language, we use the trivialization of the bundle by parallel transportation along the rays: the potential $a$ vanishes on the radial vector field. ${ }^{2}$ Here, this is simply the fact that, for any closed 2-form $B$ on $\mathbb{R}^{2}$, there exists an unique 1-form $a=\sum_{j=1}^{d} a_{j} d x_{j}$ so that $d a=B$ and $\sum_{j=1}^{d} x_{j} a_{j}=0$.

\footnotetext{
${ }^{2}$ This gauge is sometimes called the Fock-Schwinger gauge; in [Atiyah et al. 1973], it is called the synchronous framing.
} 
We will do that for the Taylor expansions degree by degree. In what follows we will use a decomposition for 1 -forms, but it works also for $k$-forms.

Let us denote by $\Omega_{N}^{k}$ the finite dimensional vector space of $k$-differential forms on $\mathbb{R}^{d}$ whose coefficients are homogeneous polynomials of degree $N$ and by $W=\sum_{j=1}^{d} x_{j} \partial / \partial x_{j}$ the radial vector field. The exterior differential induces a linear map from $\Omega_{N}^{k}$ into $\Omega_{N-1}^{k+1}$ and the inner product $\iota(W)$ a map from $\Omega_{N}^{k}$ into $\Omega_{N+1}^{k-1}$. They define complexes which are exact except at $k=N=0$. Moreover, we have a situation similar to Hodge theory:

$$
\Omega_{N}^{k}=d \Omega_{N+1}^{k-1} \oplus \iota(W) \Omega_{N-1}^{k+1} .
$$

This is due to Cartan's formula: the Lie derivative of a form $\omega \in \Omega_{N}^{k}$ satisfies, from the direct calculation, $\mathscr{L}_{W} \omega=(k+N) \omega$, and, by Cartan's formula, $\mathscr{L}_{W} \omega=d(\iota(W) \omega)+\iota(W) d \omega$. So

$$
\omega=\frac{1}{k+N}(d(\iota(W) \omega)+\iota(W) d \omega) .
$$

It remains to show that this is a direct sum: if $\omega=d \alpha=\iota(W) \gamma$, we have $\iota(W) \omega=0$ and $d \omega=0$; from the previous decomposition, we see that $\omega=0$. Let us denote by $J^{N} \omega$, where $\omega$ is a differential form of degree $k$, the form in $\Omega_{N}^{k}$ which appears in the Taylor expansion of $\omega$.

We get:

Proposition 1. If $P\left(J^{0} a, J^{1} a, \cdots, J^{N} a\right)$ is a polynomial in the Taylor expansion of the 1-form a at some order $N$ which is invariant by $a \rightarrow a+d S, P$ is independent of $J^{0} a$ and

$$
P\left(J^{1} a, \cdots, J^{N} a\right)=P\left(\frac{1}{2} J^{1} \iota(W) B, \cdots, \frac{1}{N+1} J^{N} \iota(W) B\right)
$$

is a polynomial of the Taylor expansion of $B$ to the order $N-1$.

\section{Properties of the $\boldsymbol{P}_{j, l}$}

5.1. Range of $l$ for $\boldsymbol{j}$ fixed. From the scaling properties, we deduce that, in a monomial

$$
D^{\alpha_{1}} B_{i_{1}, j_{1}} \cdots D^{\alpha_{k}} B_{i_{k}, j_{k}} D^{\beta_{1}} V \cdots D^{\beta_{m}} V
$$

belonging to $P_{j, l}$, we have $k+2 m=2(l-j)$ and $k+\left|\alpha_{1}\right|+\cdots+\left|\alpha_{k}\right|+\left|\beta_{1}\right|+\cdots+\left|\beta_{m}\right|=2 j$. Moreover, for $j \geq 1, k+m \geq 1$ and $\left|\beta_{p}\right| \geq 1$. Hence $j+1 \leq l \leq 3 j$. The previous bounds are sharp: take the monomials $\Delta^{j} V$ and $\|\nabla V\|^{2 j}$ which give $l=j+1$ and $l=3 j$.

\subsection{Invariance properties.}

(1) Let us assume that we look at the point $x=0$ and consider the operator $D_{\mu}(f)(x)=f(\mu x)$. We have

$$
D_{\mu} \circ \hat{H}_{\hbar, A, V} \circ D_{1 / \mu}=\hat{H}_{\hbar / \mu, A \circ D_{\mu}, V \circ D_{\mu}} .
$$


The same relation is true for any function $f\left(\hat{H}_{\hbar, A, V}\right)$ and then we have, looking at the Schwartz kernels and using the Jacobian $\mu^{d}$ of $D_{\mu}$ :

$$
P_{j, l}^{B, V}(0) \int_{\mathbb{R}^{d}} f^{(l)}\left(\|\xi\|^{2}+V(0)\right)|d \xi|=\mu^{-2 j} P_{j, l}^{\mu, \mu^{\star} B, \mu^{\star} V}(0) \int_{\mathbb{R}^{d}} f^{(l)}\left(\|\xi\|^{2}+V(0)\right)|d \xi| .
$$

(2) We have

$$
\widehat{H}_{\hbar, \mu a, \mu^{2} V}=\mu^{2} \widehat{H}_{\frac{\hbar}{\mu}, a, V} .
$$

(3) Changing $V$ into $V+c$ gives a translation by $c$ in the function $f$ but does not change the $P_{j, l}^{B, V}$.

(4) Changing $B$ into $-B$ gives a complex conjugation in the computations. The final result is real-valued.

(5) Orthogonal invariance is clear: an orthogonal change of coordinates around the point $x$ preserves the density of states.

5.3. The case $\boldsymbol{d}=2$. We deduce from the scaling properties and invariance by the orthogonal group, that there exists constants $a_{d}, b_{d}, c_{d}$ so that $P_{1,2}^{B, V}(x)=a_{d} \Delta V+b_{d}\|B\|^{2}, P_{1,3}(x)=c_{d}\|\nabla V\|^{2}$.

\section{Explicit examples}

The calculation for the harmonic oscillators and the constant magnetic fields allows to determine the constants $a_{d}, b_{d}, c_{d}$.

6.1. Harmonic oscillators. Let us consider $\Omega=-\hbar^{2} \frac{d^{2}}{d x^{2}}+x^{2}$ with $d=1$. The kernel of $P(t, x, y)$ of $\exp (-t \Omega)$ is given by the Mehler formula:

$$
P(t, x, y)=(2 \pi \hbar \sinh (2 t \hbar))^{-\frac{1}{2}} \exp \left(-\frac{1}{2 \hbar \sinh (2 t \hbar)}\left(\cosh (2 t \hbar)\left(x^{2}+y^{2}\right)-2 x y\right)\right) .
$$

Hence

$$
P(t, x, x) \sim(2 \pi \hbar)^{-1} e^{-t x^{2}}\left(\int_{\mathbb{R}} e^{-t \xi^{2}} d \xi\right)\left(1-\hbar^{2}\left(t^{2}-t^{3} x^{2}\right) / 3+O\left(\hbar^{4}\right)\right) .
$$

Hence $P_{1,2}(x)=-V^{\prime \prime}(x) / 6$ and $P_{1,3}(x)=-V^{\prime}(x)^{2} / 12$.

Similarly, in dimension $d>1$, we get $P_{1,2}(x)=-\Delta V(x) / 6$ and $P_{1,3}(x)=-\|\nabla V\|^{2} / 12$.

6.2. Constant magnetic field. Let us consider the case of a constant magnetic field $B$ in the plane and denote by $Q(t, x, y)$ the kernel of $\exp \left(-t H_{B, 0}\right)$. We have (see [Avron et al. 1978])

$$
Q(t, x, x)=\frac{B}{4 \pi \hbar \sinh B t \hbar} .
$$

Hence the asymptotic expansion

$$
Q(t, x, x)=(2 \pi \hbar)^{-2} \int \exp \left(-t\|\xi\|^{2}\right)|d \xi|\left(1-t^{2} \hbar^{2} B^{2} / 6+O\left(\hbar^{4}\right)\right)
$$

hence $P_{1,2}(x)=-B^{2} / 6$ and $P_{1,3}(x)=0$. 
Using the normal form $B=b_{12} d x_{1} \wedge d x_{2}+b_{34} d x_{3} \wedge d x_{4}+\cdots$, we get in dimension $d>2$ the values $P_{1,2}(x)=-\|B\|^{2} / 6$ and $P_{1,3}(x)=0$.

\section{Heat expansion from the semiclassical expansions}

We have $t \hat{H}_{1, a, V}=\widehat{H}_{\sqrt{t}, \sqrt{t} a, t V}$. Using the expansion of Theorem 1 with $f(E)=e^{-E}$, we get easily the point-wise expansion of the heat kernel on the diagonal as $t \rightarrow 0^{+}$:

$$
\left[\exp \left(-t \hat{H}_{1, a, V}\right)\right](x, x) \sim \frac{1}{(4 \pi t)^{d / 2}} e^{-t V(x)} \sum_{l=0}^{\infty}\left(\sum_{l / 3 \leq j \leq l-1} P_{j, l}^{B, V}(x)\right)(-t)^{l} .
$$

In particular, $a_{1}(x)=-V(x)$ and the coefficient $a_{2}(x)$ is given by

$$
a_{2}(x)=\frac{1}{2} V(x)^{2}-\frac{1}{6} \Delta V(x)-\frac{1}{6}\|B(x)\|^{2} .
$$

This formula agrees with Equation (3) of Theorem 3.3.1 in [Gilkey 2004].

This gives another way to compute the $P_{j, l}$ : if, as power series in $t$,

$$
\sum_{l=0}^{\infty}(-1)^{l} b_{l}(x) t^{l}=e^{t V(x)} \sum_{l=0}^{\infty} a_{l}(x) t^{l}
$$

we have

$$
\sum_{l / 3 \leq j \leq l-1} P_{j, l}^{B, V}(x)=b_{l}(x) .
$$

$P_{j, l}^{B, V}$ is the sum of monomials homogeneous of degree $2(l-j)$ in $b_{l}$ where $B$ and its derivatives have weights 1 while $V$ and its derivatives have weights 2 .

The heat coefficients $a_{l}$ on flat spaces are known for $l \leq 6$ from [Ven 1998]. This is enough to check the term in $\hbar^{2}$ (uses $a_{2}$ and $a_{3}$ ) in [Helffer and Purice 2010] and to compute the term in $\hbar^{4}$ in the semiclassical expansion (uses the $a_{l}$ for $3 \leq l \leq 6$ ).

We have also a mixed expansion writing $t \widehat{H}_{\hbar, a, V}=\widehat{H}_{\sqrt{t} \hbar, \sqrt{t} a, t V}$, we get a power series expansion in powers of $\hbar$ and $t$ valid in the domain $\hbar^{2} t \rightarrow 0$ and $0<t \leq t_{0}$ for the point-wise trace of $\exp \left(-t \widehat{H}_{\hbar, a, V}\right)$ :

$$
Z_{t, \hbar}(x) \sim \frac{1}{(4 \pi t)^{d / 2}} e^{-t V(x)}\left(1+\sum_{\substack{j \geq 1 \\ j+1 \leq l \leq 3 j}} \hbar^{2 j}(-t)^{l} P_{j, l}^{B, V}(x)\right) .
$$

This shows that the integrals $\int_{X} V(x)^{k}|d x|$ and $\int_{X} P_{j, l}^{B, V}(x)|d x|$ are recoverable from the semiclassical spectrum.

\section{Appendix: functional calculus in domains and self-adjoint extensions (after Johannes Sjöstrand)}

The content of this Appendix is due to Johannes Sjöstrand. I thank him very much for this contribution.

Let $X \subset \mathbb{R}^{d}$ be an open set. We say that a linear operator $A$ is a $\Psi D O$ in $X$, with Weyl symbol $a$ if, for any compact $K \subset X, A$ acts on functions supported in $K$ as a $\Psi D O$ of Weyl symbol $a$. 
Theorem 2. Let $H_{\hbar, a, V}$ be a Schrödinger operator with magnetic field given by

$$
H_{\hbar, a, V}=\sum_{j=1}^{d}\left(\frac{\hbar}{i} \frac{\partial}{\partial x_{j}}-a_{j}(x)\right)^{2}+V(x),
$$

defined in some open domain $X \subset \mathbb{R}^{d}$. We assume that $a$ and $V$ are smooth in $X$ and that $V$ is bounded from below, so that $H_{\hbar, a, V}$ admits some self-adjoint extensions on the Hilbert space $L^{2}(X,|d x|)$. One of them will be denoted by $\hat{H}_{\hbar, a, V}$. Then, for any $f \in \mathscr{S}(\mathbb{R}), f\left(\hat{H}_{\hbar, a, V}\right)$, given by the functional calculus, is a semiclassical $\Psi D O$ in $X$ whose symbol is given by Equation (1) and is independent of the chosen extension.

The proof uses a multicommutator method already used by Helffer and Sjöstrand [1984].

Proof. We introduce, for $s \in \mathbb{R}$, the semiclassical ( $\hbar$-dependent) Sobolev spaces

$$
\mathscr{H}_{\hbar}^{s}:=\left\{u \in \mathscr{Y}^{\prime}\left(\mathbb{R}^{d}\right) \mid\left\|\mathrm{Op}_{\hbar}\left(1+\|\xi\|^{2}\right)^{s / 2} u\right\|_{L^{2}}<\infty\right\}
$$

with the norm

$$
\|u\|_{s}:=\left\|\mathrm{Op}_{\hbar}\left(1+\|\xi\|^{2}\right)^{s / 2} u\right\|_{L^{2}}
$$

The ( $\hbar$-dependent) norm $\|A\|_{s_{1}, s_{2}}$ is the norm of $A$ as linear operator from $\mathscr{H}_{\hbar}^{s_{1}}$ to $\mathscr{H}_{\hbar}^{s_{2}}$. A linear operator $K$ is smoothing if, for all $s_{1}, s_{2},\|K\|_{s_{1}, s_{2}}=O\left(\hbar^{\infty}\right)$. This implies that the Schwartz kernel of $K$ is smooth with all derivatives locally $O\left(\hbar^{\infty}\right)$. We have the

Lemma 1. Let $Y$ be an open set in $\mathbb{R}^{d}$. Let $P_{j}=P_{j}(\hbar), j=0,1$ be two self-adjoint operators on Hilbert spaces $\mathscr{H}_{j}=L^{2}\left(X_{j},|d x|\right)$ with $Y \Subset X_{0} \subseteq X_{1} \subseteq \mathbb{R}^{d}$ and with domains $\mathscr{D}_{j}$ so that $C_{o}^{\infty}(Y) \subset \mathscr{D}_{j} \subset \mathscr{H}_{j}$. Let us assume that, on $C_{o}^{\infty}(Y), P_{0}=P_{1}=H_{\hbar, a, V}(=P)$.

Then, for any $f \in C_{o}^{\infty}(\mathbb{R}), f\left(P_{0}\right)-f\left(P_{1}\right)$ is smoothing on $Y$. In particular, the densities of states $\left[f\left(P_{j}\right)\right](x, x), j=0,1$, coincide in $Y$ modulo $O\left(\hbar^{\infty}\right)$.

Assuming Lemma 1, Theorem 2 follows by extending $a$ and $V$ smoothly outside $Y$ so that they have compact support in $\mathbb{R}^{d}$. We take $Y \Subset X=X_{0} \subset \mathbb{R}^{d}=X_{1}$. It follows that $P_{1}$ is essentially self-adjoint and the functional calculus for $P_{1}$ follows then easily from [Helffer and Robert 1983]. The result is valid even for $f \in \mathscr{S}(\mathbb{R})$ because $C_{o}^{\infty}$ is dense in $\mathscr{Y}$ and the result of [Helffer and Robert 1983] is valid for $f \in \mathscr{S}$ and the resulting formulas for the symbols are continuous w.r. to the topology of $\mathscr{Y}$.

Proof. Proof of Lemma 1 If $\chi \in C_{o}^{\infty}(Y)$, then, for $z \notin \mathbb{R}$ and $j, k \in\{0,1\}$, we have on $L^{2}(Y)$ :

$$
\left(P_{j}-z\right)^{-1} \circ \chi=\chi \circ\left(P_{k}-z\right)^{-1}-\left(P_{j}-z\right)^{-1}[P, \chi]\left(P_{k}-z\right)^{-1}
$$

Let $\chi_{0} \leq \chi_{1} \leq \cdots \leq \chi_{N}$ with, for $l=0, \cdots, N, \chi_{l} \in C_{o}^{\infty}(Y)$ and, for $l=0, \cdots, N-1, \chi_{l}\left(1-\chi_{l+1}\right) \equiv 0$. By iterating (2) and using $\chi_{l+1}\left[P, \chi_{l}\right]=\left[P, \chi_{l}\right]$, we find:

$$
\begin{gathered}
\left(P_{1}-z\right)^{-1} \circ \chi_{0}=\chi_{1} \circ\left(P_{0}-z\right)^{-1} \chi_{0}-\chi_{2} \circ\left(P_{0}-z\right)^{-1}\left[P, \chi_{1}\right]\left(P_{0}-z\right)^{-1} \chi_{0}+\cdots \\
\quad \pm \chi_{N}\left(P_{0}-z\right)^{-1}\left[P, \chi_{N-1}\right]\left(P_{0}-z\right)^{-1}\left[P, \chi_{N-2}\right] \cdots\left(P_{0}-z\right)^{-1} \chi_{0} \\
\mp\left(P_{1}-z\right)^{-1}\left[P, \chi_{N}\right]\left(P_{0}-z\right)^{-1} \cdots\left(P_{0}-z\right)^{-1} \chi_{0}
\end{gathered}
$$


Let us give now $\chi_{0}, \psi \in C_{o}^{\infty}(Y)$ with disjoints supports. By choosing the $\chi_{l}$ for $l>0$ with supports disjoint from the support of $\psi$, we see, using Equation (2), that, for any $N$,

$$
\left\|\psi\left(P_{1}-z\right)^{-1} \chi_{0}\right\|_{0,2}=O\left(\hbar^{N}|\mathfrak{s} z|^{-(N+1)}\right) .
$$

The standard a priori elliptic estimates

$$
\|u\|_{s+2, \Omega_{1}} \leq C\left(\|(P-z) u\|_{s, \Omega_{2}}+\|u\|_{s, \Omega_{1}}\right)
$$

for $z \in K \Subset \mathbb{C}$ and $\Omega_{1} \Subset \Omega_{2} \Subset \mathbb{R}^{d}$, allow to prove that, for any $N$, s, there exists $M(N, s)$ so that

$$
\left\|\psi\left(P_{1}-z\right)^{-1} \chi_{0}\right\|_{s, s+N+2}=O\left(\hbar^{N}|\Im z|^{-M(N, s)}\right)
$$

Let $\chi \in C_{o}^{\infty}(Y)$ so that $\chi \equiv 1$ on the support of $\chi_{0}$. Let us apply multiplication by $\chi_{0}$ to the right and to the left in (2) and choose $\psi$ with support disjoint from $\chi_{0}$ so that $[P, \chi] \psi=[P, \chi]$. Inserting $\psi$ this way in (2), we get, using (3),

$$
\chi_{0}\left(P_{1}-z\right)^{-1} \chi_{0}-\chi_{0}\left(P_{0}-z\right)^{-1} \chi_{0}=K,
$$

and, for any $N$, there exists $M(N)$ so that $\|K\|_{-N, N}=O\left(\hbar^{N} \Im_{z}-M(N)\right)$. We now apply the formula (known to some people as the "Helffer-Sjöstrand formula", proved for example in [Dimassi and Sjöstrand 1999, p. 94-95]), valid for $f \in C_{o}^{\infty}(\mathbb{R})$ and $\tilde{f}$ an almost holomorphic extension of $f$ :

$$
f\left(P_{j}\right)=\frac{1}{\pi} \int_{\mathbb{C}} \partial_{\bar{z}} \tilde{f}(z)\left(P_{j}-z\right)^{-1} d L(z),
$$

where $d L(z)$ is the canonical Lebesgue measure in the complex plane. From this, we see that $f\left(P_{0}\right)-$ $f\left(P_{1}\right)$ is smoothing in $Y$.

\section{Acknowledgments}

I thank the referee for his careful reading of the paper which forced me to make the statements and proofs more precise. Many thanks also to Johannes Sjöstrand for his help in clarifying some points of semiclassical analysis and for allowing me to present them in the Appendix to this paper.

\section{References}

[Atiyah et al. 1973] M. Atiyah, R. Bott, and V. K. Patodi, "On the heat equation and the index theorem”, Invent. Math. 19 (1973), 279-330. MR 58 \#31287 Zbl 0257.58008

[Avramidi 1990] I. G. Avramidi, "The covariant technique for the calculation of the heat kernel asymptotic expansion", Phys. Lett. B 238:1 (1990), 92-97. MR 91f:58092

[Avron et al. 1978] J. Avron, I. Herbst, and B. Simon, "Schrödinger operators with magnetic fields, I: General interactions", Duke Math. J. 45:4 (1978), 847-883. MR 80k:35054 Zbl 0399.35029

[Berger 1966] M. Berger, "Sur le spectre d'une variété Riemannienne”, C. R. Acad. Sci. Paris Sér. A 263 (1966), 13-16. MR 34 \#1964 Zbl 0141.38203

[Berger et al. 1971] M. Berger, P. Gauduchon, and E. Mazet, Le spectre d'une variété Riemannienne, Lecture Notes in Mathematics 194, Springer, Berlin, 1971. MR 43 \#8025 Zbl 0223.53034

[Charles 2003] L. Charles, "Berezin-Toeplitz operators: a semi-classical approach", Comm. Math. Phys. 239:1-2 (2003), 1-28. MR 2004m:53158 Zbl 1059.47030 
[Dimassi and Sjöstrand 1999] M. Dimassi and J. Sjöstrand, Spectral asymptotics in the semi-classical limit, London Mathematical Society Lecture Note Series 268, Cambridge University Press, Cambridge, 1999. MR 2001b:35237 Zbl 0926.35002

[Gilkey 1975] P. B. Gilkey, “The spectral geometry of a Riemannian manifold”, J. Differential Geom. 10:4 (1975), 601-618. MR 53 \#4150 Zbl 0316.53035

[Gilkey 2004] P. B. Gilkey, Asymptotic formulae in spectral geometry, CRC, Boca Raton, FL, 2004. MR 2005f:58041 Zbl 1080.58023

[Gracia-Saz 2005] A. Gracia-Saz, "The symbol of a function of a pseudo-differential operator", Ann. Inst. Fourier (Grenoble) 55:7 (2005), 2257-2284. MR 2006m:53142 Zbl 1091.53062

[Helffer and Purice 2010] B. Helffer and R. Purice, "Magnetic calculus and semiclassical trace formulas", J. Phys. A 43:47 (2010), 474028. MR 2011m:81089 Zbl 1204.81066 arXiv 1005.1795v1

[Helffer and Robert 1983] B. Helffer and D. Robert, "Calcul fonctionnel par la transformation de Mellin et opérateurs admissibles", J. Funct. Anal. 53:3 (1983), 246-268. MR 85i:47052 Zbl 0524.35103

[Helffer and Sjöstrand 1984] B. Helffer and J. Sjöstrand, "Multiple wells in the semiclassical limit, I", Comm. Partial Differential Equations 9:4 (1984), 337-408. MR 86c:35113 Zbl 0546.35053

[Hitrik 2002] M. Hitrik, "Existence of resonances in magnetic scattering", J. Comput. Appl. Math. 148:1 (2002), 91-97. MR 2003m:81063 Zbl 1010.81084

[Hitrik and Polterovich 2003a] M. Hitrik and I. Polterovich, "Regularized traces and Taylor expansions for the heat semigroup", J. London Math. Soc. (2) 68:2 (2003), 402-418. MR 2004h:35045 Zbl 1167.35335

[Hitrik and Polterovich 2003b] M. Hitrik and I. Polterovich, "Resolvent expansions and trace regularizations for Schrödinger operators", pp. 161-173 in Advances in differential equations and mathematical physics (Birmingham, AL, 2002), edited by Y. Karpeshina et al., Contemp. Math. 327, American Mathematical Society, Providence, RI, 2003. MR 2004g:35056 Zbl 1109.35030

[Kac 1966] M. Kac, “Can one hear the shape of a drum?", Amer. Math. Monthly 73:4, part II (1966), 1-23. MR 34 \#1121 Zbl 0139.05603

[McKean and Singer 1967] H. P. McKean, Jr. and I. M. Singer, "Curvature and the eigenvalues of the Laplacian", J. Differential Geom. 1:1 (1967), 43-69. MR 36 \#828 Zbl 0198.44301

[Polterovich 2000] I. Polterovich, "Heat invariants of Riemannian manifolds", Israel J. Math. 119 (2000), 239-252. MR 2002b: 58031 Zbl 0996.58019

[Ven 1998] A. E. M. van de Ven, “Index-free heat kernel coefficients”, Classical Quantum Gravity 15:8 (1998), 2311-2344. MR 2000a:58072 Zbl 0937.58018

Received 18 Apr 2011. Revised 11 Jun 2011. Accepted 11 Jul 2011.

YVES COLIN DE VERDIÈRE: yves.colin-de-verdiere@ujf-grenoble.fr

Unité mixte de recherche CNRS-UJF 5582, Institut Fourier, BP 74, 38402-Saint Martin d'Hères Cedex, France 


\title{
Analysis \& PDE
}

\author{
msp.berkeley.edu/apde
}

EDITORS

EDITOR-IN-CHIEF

Maciej Zworski

University of California

Berkeley, USA

BOARD OF EDITORS

\begin{tabular}{|c|c|c|c|}
\hline Michael Aizenman & $\begin{array}{l}\text { Princeton University, USA } \\
\text { aizenman@math.princeton.edu }\end{array}$ & Nicolas Burq & $\begin{array}{l}\text { Université Paris-Sud 11, France } \\
\text { nicolas.burq@math.u-psud.fr }\end{array}$ \\
\hline Luis A. Caffarelli & $\begin{array}{l}\text { University of Texas, USA } \\
\text { caffarel@ math.utexas.edu }\end{array}$ & un-Yung Alice Chang & $\begin{array}{l}\text { Princeton University, USA } \\
\text { chang@ math.princeton.edu }\end{array}$ \\
\hline Michael Christ & $\begin{array}{l}\text { University of California, Berkeley, USA } \\
\text { mchrist@math.berkeley.edu }\end{array}$ & Charles Fefferman & $\begin{array}{l}\text { Princeton University, USA } \\
\text { cf@math.princeton.edu }\end{array}$ \\
\hline Ursula Hamenstaedt & $\begin{array}{l}\text { Universität Bonn, Germany } \\
\text { ursula@math.uni-bonn.de }\end{array}$ & Nigel Higson & $\begin{array}{l}\text { Pennsylvania State Univesity, USA } \\
\text { higson@math.psu.edu }\end{array}$ \\
\hline Vaughan Jones & $\begin{array}{l}\text { University of California, Berkeley, USA } \\
\text { vfr@math.berkeley.edu }\end{array}$ & Herbert Koch & $\begin{array}{l}\text { Universität Bonn, Germany } \\
\text { koch@math.uni-bonn.de }\end{array}$ \\
\hline Izabella Laba & $\begin{array}{l}\text { University of British Columbia, Canada } \\
\text { ilaba@math.ubc.ca }\end{array}$ & Gilles Lebeau & $\begin{array}{l}\text { Université de Nice Sophia Antipolis, France } \\
\text { lebeau@unice.fr }\end{array}$ \\
\hline László Lempert & $\begin{array}{l}\text { Purdue University, USA } \\
\text { lempert@math.purdue.edu }\end{array}$ & Richard B. Melrose & $\begin{array}{l}\text { Massachussets Institute of Technology, USA } \\
\text { rbm@math.mit.edu }\end{array}$ \\
\hline Frank Merle & $\begin{array}{l}\text { Université de Cergy-Pontoise, France } \\
\text { Frank.Merle@u-cergy.fr }\end{array}$ & William Minicozzi II & $\begin{array}{l}\text { Johns Hopkins University, USA } \\
\text { minicozz@ math.jhu.edu }\end{array}$ \\
\hline Werner Müller & $\begin{array}{l}\text { Universität Bonn, Germany } \\
\text { mueller@math.uni-bonn.de }\end{array}$ & Yuval Peres & $\begin{array}{l}\text { University of California, Berkeley, USA } \\
\text { peres@stat.berkeley.edu }\end{array}$ \\
\hline Gilles Pisier & $\begin{array}{l}\text { Texas A\&M University, and Paris } 6 \\
\text { pisier@math.tamu.edu }\end{array}$ & Tristan Rivière & $\begin{array}{l}\text { ETH, Switzerland } \\
\text { riviere@math.ethz.ch }\end{array}$ \\
\hline Igor Rodnianski & $\begin{array}{l}\text { Princeton University, USA } \\
\text { irod@math.princeton.edu }\end{array}$ & Wilhelm Schlag & $\begin{array}{l}\text { University of Chicago, USA } \\
\text { schlag@math.uchicago.edu }\end{array}$ \\
\hline Sylvia Serfaty & $\begin{array}{l}\text { New York University, USA } \\
\text { serfaty@ cims.nyu.edu }\end{array}$ & Yum-Tong Siu & $\begin{array}{l}\text { Harvard University, USA } \\
\text { siu@math.harvard.edu }\end{array}$ \\
\hline Terence Tao & $\begin{array}{l}\text { University of California, Los Angeles, USA } \\
\text { tao@math.ucla.edu }\end{array}$ & Michael E. Taylor & $\begin{array}{l}\text { Univ. of North Carolina, Chapel Hill, USA } \\
\text { met@math.unc.edu }\end{array}$ \\
\hline Gunther Uhlmann & $\begin{array}{l}\text { University of Washington, USA } \\
\text { gunther@math.washington.edu }\end{array}$ & András Vasy & $\begin{array}{l}\text { Stanford University, USA } \\
\text { andras@math.stanford.edu }\end{array}$ \\
\hline an Virgil Voiculescu & $\begin{array}{l}\text { University of California, Berkeley, USA } \\
\text { dvv@ math.berkeley.edu }\end{array}$ & Steven Zelditch & $\begin{array}{l}\text { Northwestern University, USA } \\
\text { zelditch@math.northwestern.edu }\end{array}$ \\
\hline
\end{tabular}

\section{PRODUCTION}

contact@msp.org

Silvio Levy, Scientific Editor

Sheila Newbery, Senior Production Editor

See inside back cover or msp.berkeley.edu/apde for submission instructions.

The subscription price for 2012 is US \$140/year for the electronic version, and \$240/year for print and electronic. Subscriptions, requests for back issues from the last three years and changes of subscribers address should be sent to Mathematical Sciences Publishers, Department of Mathematics, University of California, Berkeley, CA 94720-3840, USA.

Analysis \& PDE, at Mathematical Sciences Publishers, Department of Mathematics, University of California, Berkeley, CA 94720-3840 is published continuously online. Periodical rate postage paid at Berkeley, CA 94704, and additional mailing offices.

APDE peer review and production are managed by EditFLOW ${ }^{\mathrm{TM}}$ from Mathematical Sciences Publishers.

PUBLISHED BY

mathematical sciences publishers

http://msp.org/

A NON-PROFIT CORPORATION

Typeset in IATEX

Copyright $(2012$ by Mathematical Sciences Publishers 


\section{ANALYSIS \& PDE}

\section{Volume $5 \quad$ No. $3 \quad 2012$}

On some microlocal properties of the range of a pseudodifferential operator of principal type 423 JENS WITTSTEN

Blow-up solutions on a sphere for the 3D quintic NLS in the energy space JUSTIN HOLMER and SVETLANA ROUDENKO

Sharp geometric upper bounds on resonances for surfaces with hyperbolic ends DAVID BORTHWICK

A vector field method approach to improved decay for solutions to the wave equation on a slowly rotating Kerr black hole JONATHAN LUK

On the Bogolyubov-Ruzsa lemma TOM SANDERS

Real analyticity away from the nucleus of pseudorelativistic Hartree-Fock orbitals

Anna Dall'Acqua, Søren Fournais, Thomas ØstergaArd Sørensen and EDGARDO STOCKMEYER

Semiclassical trace formulas and heat expansions

YVES COLIN DE VERDIÈRE 